\title{
Forecasting the flap: predictors for pediatric lower extremity trauma reconstruction
}

\author{
Kasra N. Fallah, Logan A. Konty, Brady J. Anderson, Alfredo Cepeda Jr, Grigorios A. Lamaris, \\ Phuong D. Nguyen, Matthew R. Greives \\ Division of Plastic Surgery, Department of Surgery, McGovern Medical School at the University of Texas Health Science Center at Houston, \\ Houston, TX, USA
}

\begin{abstract}
Background Predicting the need for post-traumatic reconstruction of lower extremity injuries remains a challenge. Due to the larger volume of cases in adults than in children, the majority of the medical literature has focused on adult lower extremity reconstruction. This study evaluates predictive risk factors associated with the need for free flap reconstruction in pediatric patients following lower extremity trauma.
\end{abstract}

Methods An IRB-approved retrospective chart analysis over a 5-year period (January 1, 2012 to December 31 , 2017) was performed, including all pediatric patients ( $<18$ years old) diagnosed with one or more lower extremity wounds. Patient demographics, trauma information, and operative information were reviewed. The statistical analysis consisted of univariate and multivariate regression models to identify predictor variables associated with free flap reconstruction.

Results In total, 1,821 patients were identified who fit our search criteria, of whom 41 patients (2.25\%) required free flap reconstruction, 65 patients (3.57\%) required local flap reconstruction, and 19 patients $(1.04 \%)$ required skin graft reconstruction. We determined that older age (odds ratio $[O R], 1.134 ; P=0.002)$, all-terrain vehicle accidents $(O R, 6.698$; $P<0.001)$, and trauma team activation $(O R, 2.443 ; P=0.034)$ were associated with the need for free flap reconstruction following lower extremity trauma in our pediatric population.

Conclusions Our study demonstrates a higher likelihood of free flap reconstruction in older pediatric patients, those involved in all-terrain vehicle accidents, and cases involving activation of the trauma team. This information can be implemented to help develop an early risk calculator that defines the need for complex lower extremity reconstruction in the pediatric population.

Keywords Pediatric / Trauma / Predictive factors / Lower extremity reconstruction / Free flap

\author{
Correspondence: Matthew R. Greives \\ Division of Plastic Surgery, \\ Department of Surgery, McGovern \\ Medical School at the University of \\ Texas Health Science Center at \\ Houston, 6410 Fannin Street, Suite \\ 1400, Houston, TX 77030, USA \\ Tel: +1-713-500-7275 \\ Fax: +1-713-500-7296 \\ E-mail: \\ Matthew.R.Greives@uth.tmc.edu
}

Received: April 20, $2021 \bullet$ Revised: August 13, 2021 - Accepted: September 6, 2021

pISSN: 2234-6163 • elSSN: 2234-6171 • https://doi.org/10.5999/aps.2021.00675• Arch Plast Surg 2022;49:91-98

\begin{abstract}
A portion of this manuscript was presented at the Texas Society of Plastic Surgeons, November 2019, in Galveston, TX, USA.
\end{abstract}

\section{INTRODUCTION}

Trauma remains the leading cause of mortality in the United States and the overall impact of pediatric trauma extends even beyond mortality. Nonfatal traumatic injuries account for over 10 million emergency department visits and over 300,000 hospitalizations annually for pediatric patients at a cost of 23 billion dollars annually [1-4]. Lower extremity trauma represents a sig- 
nificant portion of this patient population; nonetheless, research on the relevant mechanisms and reconstructive algorithms remains relatively sparse.

These young patients present a unique challenge as both the initial and definitive management can affect functional and aesthetic outcomes as they develop. A multidisciplinary evaluation at the time of patient presentation, including examination by a reconstructive team, is essential for optimizing patient outcomes. Lower extremity trauma has been extensively evaluated in adults, and numerous studies have been devised to engineer predictive models for outcomes in adult trauma [5]. The mechanism, location, and severity of injury can affect the method and timing of repair, which significantly impact patient outcomes [6]. All of these factors are important as it has been well established that early involvement of plastic surgery for flap reconstruction is associated with improved outcomes [7]. However, literature concerning these injuries in the pediatric population has not been as widely reported.

Our institution is considered one of the busiest level 1 pediatric trauma centers in the United States. Additionally, it represents a diverse area that encompasses a large array of trauma from both urban and rural settings. Given our trauma center's experience, we set out to examine these critical injuries in order to develop a predictive model of the need for complex lower extremity reconstruction to drive treatment plans in this important subset of patients. A historical challenge to this analysis has been the limited number of pediatric patients meeting review criteria in order to delineate patterns of indications for these injuries. The high volume of patients treated at our pediatric trauma center has allowed us to review a large number of pediatric patients who suffered lower extremity trauma in order to identify patient statistics and injury patterns that would aid in stratifying patients into higher-risk groups that require more complex or free flap reconstruction.

\section{METHODS}

\section{Study design}

Following institutional review board approval (IRB No. HSCMS-19-0038), we performed a retrospective chart analysis of pediatric trauma patients found in our institution's trauma registry using the International Classification of Diseases 9th Revision (ICD-9) codes 820-829, 890-897, 916, 917, 924, and 928 to identify all patients who presented with one or more lower extremity wounds between January 01, 2012 and December 31, 2017. The trauma registry is designed to prospectively capture all patients who qualify as trauma patients, although it may potentially exclude patients who entered the hospital system via al- ternative pathways. In order to study lower extremity trauma in the pediatric population, a database was created from the patients identified by the above query, requiring patient age to be less than 18 years old. Patients who died prior to a definitive record of a lower extremity wound were excluded.

\section{Data abstracted}

Patient demographics, trauma type, trauma team activation, injury severity score, and mechanism of injury data were obtained from our chart analysis. Trauma was stratified as blunt versus penetrating, as well as by the type of injury (aggravated assault, all-terrain vehicle accident, bicycle, fall, motorcycle accident, motor vehicle collision, pedestrian, sporting injury, and others). Patients' socioeconomic status was estimated based on the median income of their zip code by using the 2019 United States Census dataset. For patients with concurrent orthopedic injuries, surgical-specific variables such as the Gustilo grade, wound size, time to definitive fixation, and time to definitive reconstruction were included. The reconstructive portion for patients who required free flap reconstruction was also assessed to include the number of procedures before reconstruction and the type of free flap.

The care of pediatric patients following lower extremity trauma involved management by the pediatric, orthopedic, and plastic surgery teams. Primary wound closure was usually performed by the pediatric surgery team. However, the decisionmaking for complex reconstructive procedures was generally executed by the plastic surgery team (Fig. 1). If multiple forms of complex reconstruction (skin graft, local flap, or free flap) were performed on a patient, he or she was categorized based on the most complex procedure that was performed according to the reconstructive ladder. Free flap reconstruction was considered more complex than local flap reconstruction, and local flap reconstruction was considered more complex than skin graft reconstruction.

The primary outcome analyzed in this study was whether or not a pediatric patient received free flap reconstruction following lower extremity trauma. All patients were stratified into two groups: patients who underwent free flap reconstruction and patients who did not. The group with patients who did not undergo free flap reconstruction included those who required primary repair of lacerations, skin grafts, or local flaps.

\section{Statistical analysis}

Univariate logistic regression was conducted to evaluate the risk factors associated with the need for free flap reconstruction following lower extremity trauma in our patient population. Variables with a P-value $<0.30$ in the univariate regression analysis 

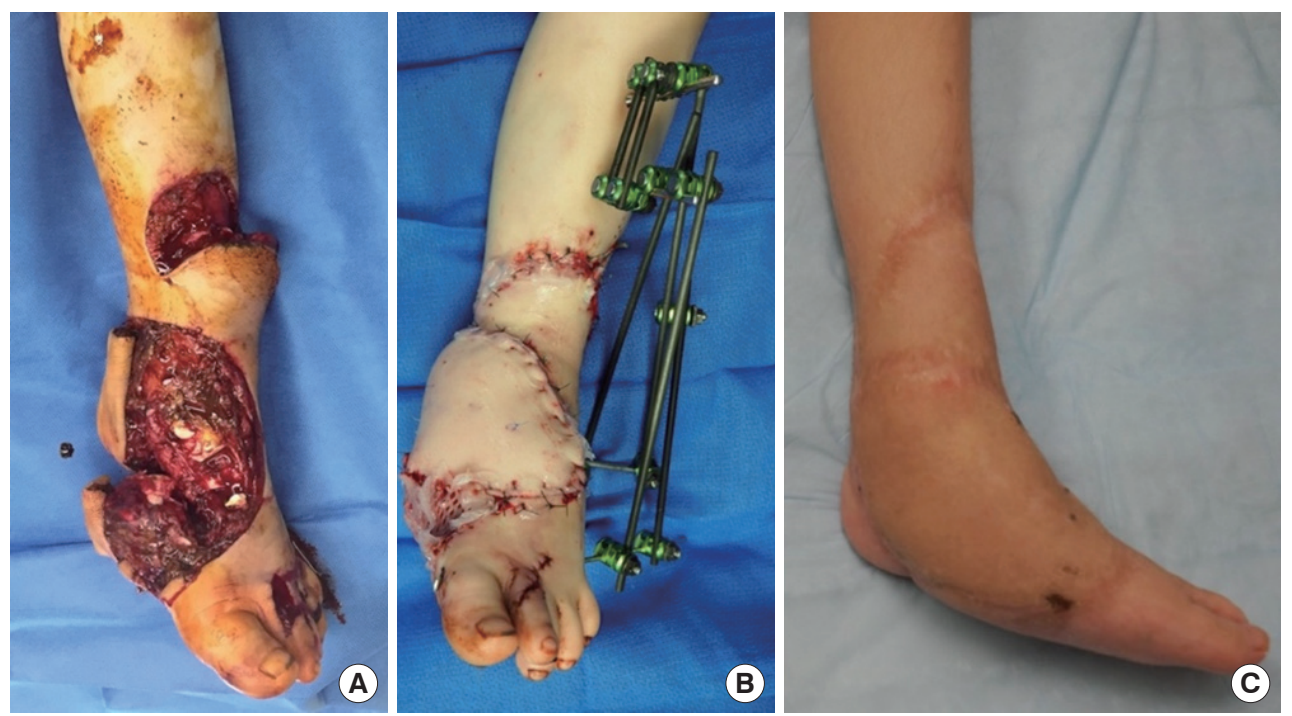

Fig. 1. A 4-year-old male with a Gustilo IIIB injury from a lawnmower. (A) Open dorsal and medial wound with exposed bone. (B) Immediately postoperative view following anterolateral thigh free flap reconstruction. (C) Two years postoperative view.

were included in a multivariate logistic regression model. A Pvalue $<0.05$ was considered to indicate statistical significance in both the univariate and the multivariate analyses. A receiver operating characteristic curve was generated to assess the performance of our multivariate model. All statistical analyses were conducted using RStudio statistical software (Boston, MA, USA).

\section{RESULTS}

\section{Patient demographics}

In total, 1,821 patients met our inclusion criteria (Fig. 2). In this patient population, 41 patients $(2.3 \%)$ underwent free flap reconstruction, 65 patients (3.6\%) underwent local flap reconstruction, and 19 patients (1.0\%) only underwent skin graft reconstruction. The median age was 10 years (interquartile range [IQR], 4-14 years) and $67 \%$ were male (Table 1 ). The most common ethnicities were non-Hispanic Caucasian patients (36\%) and Hispanic patients (29\%).

In the cohort of patients who required free flap reconstruction, the median age was 14 years (IQR, 11-15 years), which differed significantly from that of our total patient population (14 years vs. 10 years; $P<0.001)$, and $78 \%$ were male. The most common ethnicities in this cohort were non-Hispanic Caucasian patients (44\%) and Hispanic patients (24\%), which was similar to the distribution in our total patient population.

\section{Trauma information}

In our total patient population, blunt trauma occurred in $94 \%$ of patients and penetrating trauma occurred in $6 \%$ of patients, with

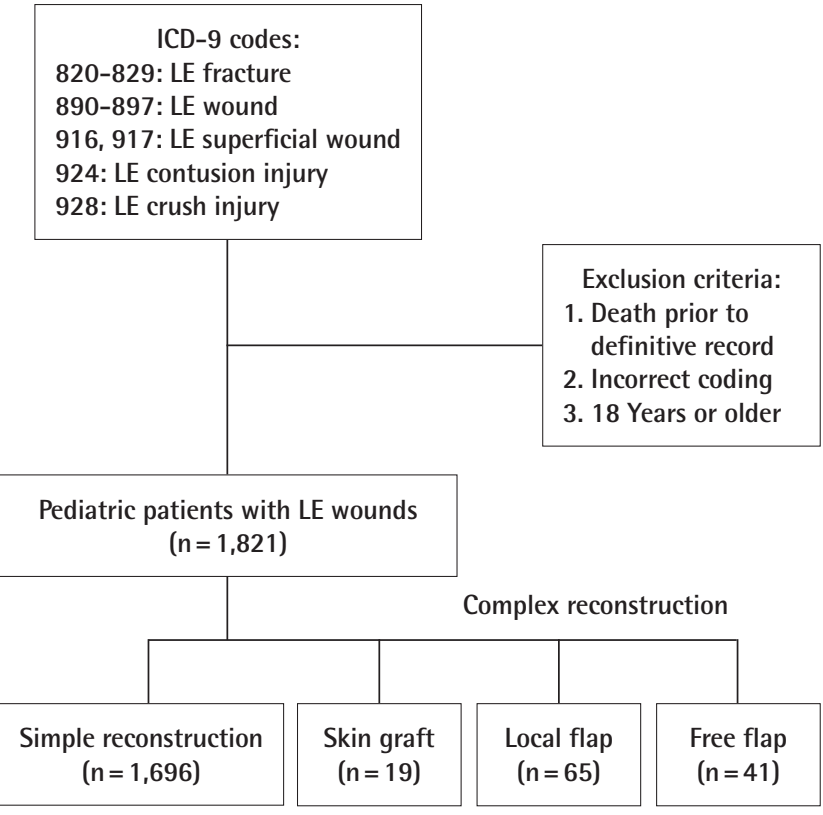

Fig. 2. International Classification of Diseases (ICD) codes used and study design. LE, lower extremity.

a median injury severity score of 9 (IQR, 4-14) (Table 2). The most common mechanisms of injury were motor vehicle collisions $(26 \%)$ and falls $(22 \%)$. The trauma team was activated in $49 \%$ of patients.

In the cohort of patients who required free flap reconstruction, blunt trauma occurred in $93 \%$ of patients and penetrating trauma occurred in $7 \%$ of patients, with a median injury severity score of 10 (IQR, 5-17). The most common mechanisms of injury in this cohort were all-terrain vehicle accidents $(24 \%)$ and motor vehicle collisions (22\%), which differed significantly 
Table 1. Patient demographics stratified by type of reconstruction

\begin{tabular}{|c|c|c|c|c|c|c|}
\hline Variable & $\begin{array}{c}\text { Total } \\
(\mathrm{n}=1,821)\end{array}$ & $\begin{array}{l}\text { Simple reconstruction } \\
\quad(n=1,696)\end{array}$ & $\begin{array}{l}\text { Skin graft } \\
(n=19)\end{array}$ & $\begin{array}{l}\text { Local flap } \\
(n=65)\end{array}$ & $\begin{array}{c}\text { Free flap } \\
(n=41)\end{array}$ & P-value \\
\hline Age (yr) & $10(4-14)$ & $10(4-14)$ & $13(9-16)$ & $14(8-16)$ & $14(11-15)$ & $<0.001^{\text {a) }}$ \\
\hline Male sex & $1,222(67)$ & $1,132(67)$ & $14(74)$ & $44(68)$ & $32(78)$ & 0.13 \\
\hline Ethnicity/race & & & & & & 0.74 \\
\hline African American & $438(24)$ & $414(24)$ & $6(32)$ & $10(15)$ & $8(20)$ & \\
\hline Asian & $21(1)$ & $19(1)$ & 0 & $1(2)$ & $1(2)$ & \\
\hline Caucasian & $654(36)$ & $604(36)$ & $5(26)$ & 27 (42) & $18(44)$ & \\
\hline Hispanic & $526(29)$ & $493(29)$ & $6(32)$ & $17(26)$ & $10(24)$ & \\
\hline Other & $182(10)$ & $166(10)$ & $2(10)$ & $10(15)$ & $4(10)$ & \\
\hline Income by zip code $(\$)$ & $\begin{array}{c}52,283 \\
(40,795-70,815)\end{array}$ & $\begin{array}{c}52,269 \\
(40,679-70,326)\end{array}$ & $\begin{array}{c}54,786 \\
(46,311-76,387)\end{array}$ & $\begin{array}{c}56,760 \\
(44,386-73,466)\end{array}$ & $\begin{array}{c}49,923 \\
(40,693-66,775)\end{array}$ & 0.22 \\
\hline
\end{tabular}

Values are presented as median (interquartile range) or number (\%).

a) Statistically significant, $\mathrm{P}<0.05$.

Table 2. Trauma information stratified by type of reconstruction

\begin{tabular}{|c|c|c|c|c|c|c|}
\hline Variable & $\begin{array}{c}\text { Total } \\
(\mathrm{n}=1,821)\end{array}$ & $\begin{array}{l}\text { Simple reconstruction } \\
\quad(n=1,696)\end{array}$ & $\begin{array}{l}\text { Skin graft } \\
(n=19)\end{array}$ & $\begin{array}{l}\text { Local flap } \\
(n=65)\end{array}$ & $\begin{array}{l}\text { Free flap } \\
(n=41)\end{array}$ & P-value \\
\hline Trauma type & & & & & & 0.72 \\
\hline Blunt & $1,712(94)$ & $1,600(94)$ & $17(89)$ & $57(88)$ & $38(93)$ & \\
\hline Penetrating & $109(6)$ & $96(6)$ & $2(11)$ & $8(12)$ & $3(7)$ & \\
\hline Trauma team activation & $886(49)$ & $800(47)$ & $9(47)$ & $45(69)$ & $32(78)$ & $<0.001^{\mathrm{a}}$ \\
\hline Median ISS & $9(4-14)$ & $9(4-13)$ & $4(1-16)$ & $9(5-16)$ & $10(5-17)$ & 0.28 \\
\hline Mechanism of injury & & & & & & $<0.001^{\mathrm{a}}$ \\
\hline Aggravated assault & $106(6)$ & $97(6)$ & $1(5)$ & $6(9)$ & $2(5)$ & \\
\hline All-terrain vehicle & $95(5)$ & $72(4)$ & $4(21)$ & $9(14)$ & $10(24)$ & \\
\hline Bicycle & $69(4)$ & $66(4)$ & 0 & $1(2)$ & $2(5)$ & \\
\hline Fall & $394(22)$ & $387(23)$ & $1(5)$ & $5(8)$ & $1(2)$ & \\
\hline Motorcycle & $42(2)$ & $32(2)$ & $1(5)$ & $5(8)$ & $4(10)$ & \\
\hline Motor vehicle & $473(26)$ & $449(26)$ & $3(16)$ & $12(18)$ & $9(22)$ & \\
\hline Pedestrian & $214(12)$ & $188(11)$ & $5(27)$ & $13(20)$ & $8(20)$ & \\
\hline Sporting & $143(8)$ & $140(8)$ & $1(5)$ & $2(3)$ & 0 & \\
\hline Other & $285(15)$ & $265(16)$ & $3(16)$ & $12(18)$ & $5(12)$ & \\
\hline
\end{tabular}

Values are presented as number (\%) or median (interquartile range).

a)Statistically significant, $\mathrm{P}<0.05$.

from the mechanisms in our total patient population $(\mathrm{P}<$ 0.001 ). The trauma team was activated in $78 \%$ of patients who required free flap reconstruction, which also differed significantly from the proportion in our total patient population ( $78 \%$ vs. 49\%; P $<0.001$ ).

\section{Operative information}

Patients who required free flap reconstruction following lower extremity trauma had a median wound size of $200 \mathrm{~cm}^{2}$ (IQR, $150-400 \mathrm{~cm}^{2}$ ), and $44 \%$ of them sustained a Gustilo grade III tibia fracture (Table 3). The median time to definitive fixation was 4 days (IQR, 1-8 days) and the median time to definitive reconstruction was 8 days (IQR, 5-9 days). The median number of procedures until free flap reconstruction was 4 (IQR, 4-6). The most common free flaps used were from the latissimus dorsi muscle (51\%) and the radial forearm (29\%).
Table 3. Operative information for patients who required free flap reconstruction

\begin{tabular}{lc}
\hline Variable & Free flap $(\mathrm{n}=41)$ \\
\hline Gustilo grade III tibia fracture & $18(44)$ \\
Wound size $\left(\mathrm{cm}^{2}\right)$ & $200(150-400)$ \\
Time to definitive fixation (day) & $4(1-8)$ \\
Time to definitive reconstruction (day) & $8(5-9)$ \\
No. of procedures until reconstruction & $4(4-6)$ \\
Type of free flap & \\
Gracilis & $2(5)$ \\
Lateral arm & $2(5)$ \\
Latissimus dorsi & $21(51)$ \\
Radial forearm & $12(29)$ \\
Rectus abdominis & $4(10)$ \\
\hline
\end{tabular}

Values are presented as number (\%) or median (interquartile range).

\section{Multivariate analysis}

Age, male sex, median income, trauma team activation, injury severity score, and mechanism of injury were included in our mul- 


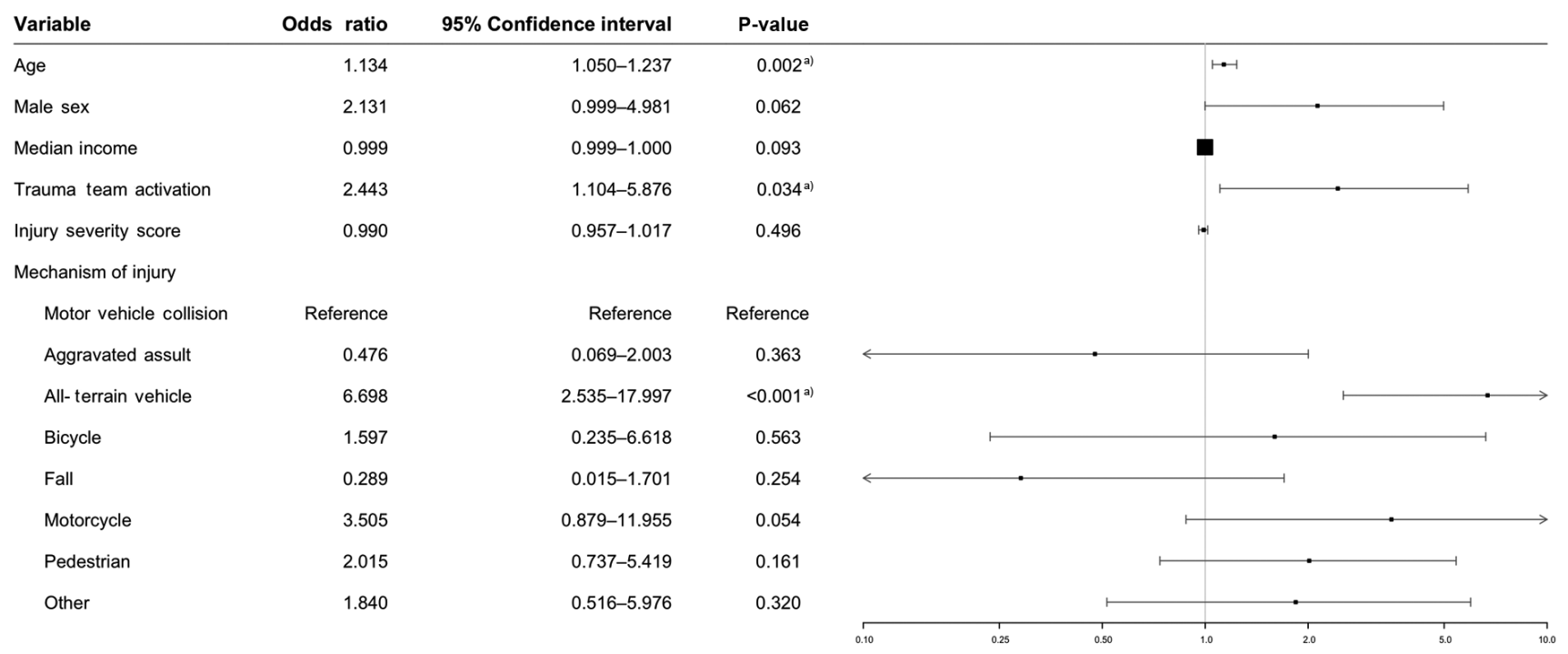

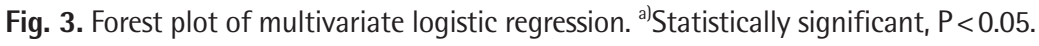

tivariate logistic regression model (Fig. 3). This analysis demonstrated that increased age (odds ratio [OR], 1.134; 95\% confidence interval $[\mathrm{CI}], 1.050-1.237 ; \mathrm{P}=0.002)$, all-terrain vehicle accidents (OR, 6.698; 95\% CI, 2.535-17.997; P < 0.001), and activation of the trauma team (OR, 2.443; 95\% CI, 1.1045.876; $\mathrm{P}=0.034$ ) remained significantly associated with an increased need for free flap reconstruction following lower extremity trauma in our pediatric population. Both male sex (OR, 2.131; 95\% CI, 0.999-4.981; P=0.062) and motorcycle accidents (OR, 3.505; 95\% CI, 0.879-11.955; P = 0.054) were positively associated with a need for free flap reconstruction in the univariate analysis, but this relationship was not maintained in the multivariate analysis. A receiver operating characteristic curve of our multivariate model generated an area under the curve of 0.84 (Fig. 4).

\section{DISCUSSION}

Lower extremity reconstruction in the pediatric population requires the utilization of all rungs of the reconstructive ladder. Classically, the Gustilo classification system helped guide both the orthopedic intervention in bony trauma and the need for soft tissue reconstruction. However, as advances in microsurgery and the utilization of free flaps have increased the complexity of repair, more detailed assessment of the initial injury is mandated. It is understood that establishing the extent of injury early in presentation helps shorten the time to reconstruction, which has been shown to reduce complications and improve functional outcomes [8]. The arterial supply also has a significant impact on complication rates following free flap reconstruction [9]. However, because lower extremity trauma can be

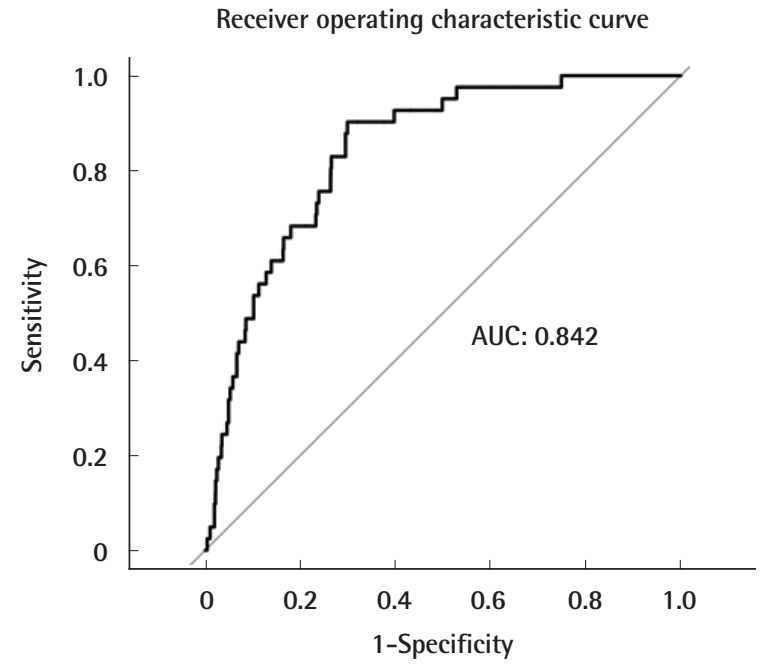

Fig. 4. Receiver operating characteristic curve for our predictive model. AUC, area under the curve.

very complex, many centers have worked to characterize in further detail other aspects of these injuries in the adult population [10]. Similar attempts have been made in the pediatric population, but there is still a paucity of data compared to the literature on adults.

Our data help establish criteria that can be identified at the time of presentation to help predict the need for higher-order reconstruction. It has been suggested that previous scoring systems may inaccurately predict long-term outcomes [11]. The Mangled Extremity Severity Score, which has been considered in both the adult and pediatric populations, took a global approach in establishing amputation likelihood with nearly $100 \%$ reported accuracy [12]. Different systems have taken a more focused approach to the assessment of lower extremity damage 
and long-term effects [13-16]. However, our data show that other factors can be incorporated into the early assessment in order to guide treatment and improve patient outcomes.

We found a male predominance in our patient population, similar to that reported in the literature on adults [17]. We also established that motor vehicle collisions accounted for the highest percentage of lower extremity trauma at our institution. Compared with adult patients, in whom motorcycle collisions were associated with the need for operative intervention for their low extremity injury, all-terrain vehicle accidents were strongly associated with free flap reconstruction in our pediatric population [18]. The mechanisms of injury seen in our pediatric patients (all-terrain vehicle accidents, falls, motor vehicle collisions, etc.) seem to be more related to recreational activities, whereas adult patients often fall victim to industrial and workrelated accidents in addition to motor vehicle collisions. Utilization of the level 1 trauma activation system was noted in nearly half of the lower extremity trauma cases at our institution. However, this system was activated in a greater proportion of patients who eventually required free flap reconstruction. Older age was also a predictor of more advanced reconstruction. Finally, blunt trauma was the cause of lower extremity injury in the majority of our cases.

Aside from injury severity, several factors can influence the success of reconstruction in these patients. Godina established the efficacy of early reconstruction, and this practice has been utilized since his publication in the 1980s [7]. Re-evaluations of this study have continued to reinforce this concept, although with some increased leniency, since most centers consider operation within 10 days of injury as "early" [19]. The location of the injury also has important implications for operative planning. Although distal reconstruction calls for more technically challenging vascular anastomoses, advances in microsurgery have enabled similarly successful results with distal anastomosis $[20,21]$. Specifically, in the pediatric population, it has been theorized that the underdeveloped muscularis layer of arterial vessels can contribute to vasospasm and flap complications [22]. This may explain why age has been shown to be a positive predictor of flap success [23]. Pediatric patients also pose unique considerations in reconstruction. Depending on age, the ratio of the lower extremity size to the rest of the body may alter flap choice and availability. Additionally, although pediatric vessels may be more prone to vasospasm, they do not incur the native arteriosclerosis and pre-existing vascular unreliability that adult patients may have. Lastly, one must consider the compliance of pediatric patients with flap protocols. Useful adjuncts to improve flap success include the use of "kickstand" external fixators and casting to prevent inadvertent positional flap compro- mise.

In the literature, complication and failure rates after reconstruction for lower extremity trauma have remained high compared with reconstruction of the upper extremity and other areas. Even with satisfactory operative technique, patient-rated results showed that a significant number of patients still need assistance with ambulation 3 months after reconstruction, and a significant proportion of patients who undergo reconstruction for lower extremity trauma have markedly lower quality of life ratings $[17,24]$. Taken together, the data point towards areas of potential improvement in the overall care of lower extremity injuries.

Limitations of this study include its retrospective design, with an evaluation of demographic and pathologic information only available through chart review. Medical records for longer-term follow-up were not available to us as they were largely from outside medical record databases. Additionally, some patients may have received different treatment pathways depending on the surgeon's evaluation because variations in surgical decisionmaking for the need for free flap reconstruction or other types of complex reconstruction were not standardized across surgeons. However, it can be difficult to objectively analyze surgeon's preferences. We also utilized a trauma database that may have missed patients due to coding issues or omission by error. Nevertheless, we decided to use this database in its entirety to avoid biasing our data by including patients from other databases that were not included in ours. Finally, this single-center study was conducted at a high-volume, academic trauma institution and the findings of this study may not be directly applicable to other institutions.

Although soft tissue injury, open fractures with exposed bone, exposed neurovascular structures, wound location, wound evolution over time, and infection can be predictive of the need for free flap reconstruction, this paper was written with a different focus. Our strategy was to analyze various sociodemographic factors in addition to information about the cause and severity of each patient's trauma. However, some of the operative factors were evaluated for patients who required free flap reconstruction purely to provide a descriptive analysis of this patient population. Unfortunately, we only received a portion of the relevant data for free flap patients and not all patients who underwent lower extremity reconstruction following trauma, so further statistical analyses beyond descriptive statistics were not carried out. We plan to investigate and analyze those factors in further research to augment the value of the information gained from this study. Additionally, we did not approach this paper with the goal of analyzing free flap reconstruction outcomes or complications. We decided to evaluate only specific predictive factors 
that were associated with the need for free flap reconstruction following lower extremity trauma. Although only 41 patients required free flap reconstruction in this study, our statistical analysis included all 1,821 cases of lower extremity trauma.

Future research at our center will further delineate details of patient presentations and outcomes that will help develop accurate and predictive algorithms for this subset of trauma patients. We have also established a rich database from which detailed information on pediatric trauma patients can be drawn. Information including success rates based on variation in injury patterns and repair types can be extracted from this database in the future.

Based on a review of trauma cases that occurred at our institution, one of the busiest level 1 pediatric trauma centers in the United States, lower extremity trauma in the pediatric population poses a necessary call to action for further research. Previous studies have shown that early reconstruction in these patients is related to better results. Using the factors outlined in this study, institutions can help identify these pediatric patients at the time of their presentation to their trauma facility in order to improve their functional and aesthetic outcomes. Smaller, outlying institutions can also use these statistics as a guide for patients who may present to their facility but require transfer to a tertiary center in order to ensure successful outcomes for these young patients. Free flap reconstruction will continue to be a treatment option in the pediatric population, and our study aims to improve early identification and referral to pediatric reconstruction specialists $[25,26]$. Therefore, we believe that our work in identifying the patients who will benefit the most from complex surgical reconstruction can significantly improve outcomes for pediatric trauma patients.

\section{NOTES}

\section{Conflict of interest}

No potential conflict of interest relevant to this article was reported.

\section{Ethical approval}

The study was approved by the Institutional Review Board of Memorial Hermann Hospital (IRB No. HSC-MS-19-0038) and performed in accordance with the principles of the Declaration of Helsinki. The informed consent was waived because this study design is a retrospective chart review.

\section{Patient consent}

The patients provided written informed consent for the publication and the use of their images.

\section{Author contribution}

Conceptualization: KN Fallah. Data curation: KN Fallah, BJ Anderson. Formal analysis: KN Fallah. Methodology: KN Fallah. Project administration: PD Nguyen, MR Greives. Writing original draft: KN Fallah. Writing - review \& editing: KN Fallah, LA Konty, A Cepeda Jr, GA Lamaris, PD Nguyen, MR Greives. All authors read and approved the final manuscript.

\section{ORCID}

Kasra N. Fallah https://orcid.org/0000-0002-1285-5669

Logan A. Konty https://orcid.org/0000-0002-4225-5605

Brady J.Anderson https://orcid.org/0000-0002-6955-797X

Alfredo Cepeda Jr https://orcid.org/0000-0003-0772-2017 Grigorios A. Lamaris https://orcid.org/0000-0002-0099-2041

Phuong D. Nguyen https://orcid.org/0000-0002-2116-902X Matthew R. Greives https://orcid.org/0000-0003-4602-5054

\section{REFERENCES}

1. Hambidge SJ, Davidson AJ, Gonzales R, et al. Epidemiology of pediatric injury-related primary care office visits in the United States. Pediatrics 2002;109:559-65.

2. Dowd MD, Keenan HT, Bratton SL. Epidemiology and prevention of childhood injuries. Crit Care Med 2002;30(11 Suppl):S385-92.

3. Avraham JB, Bhandari M, Frangos SG, et al. Epidemiology of paediatric trauma presenting to US emergency departments: 2006-2012. Inj Prev 2019;25:136-43.

4. Javali RH, Krishnamoorthy, Patil A, et al. Comparison of Injury Severity Score, New Injury Severity Score, Revised Trauma Score and Trauma and Injury Severity Score for mortality prediction in elderly trauma patients. Indian J Crit Care Med 2019;23:73-7.

5. Bosse MJ, MacKenzie EJ, Kellam JF, et al. A prospective evaluation of the clinical utility of the lower-extremity injury-severity scores. J Bone Joint Surg Am 2001;83:3-14.

6. Gopal S, Giannoudis PV, Murray A, et al. The functional outcome of severe, open tibial fractures managed with early fixation and flap coverage. J Bone Joint Surg Br 2004;86: 861-7.

7. Godina M. Early microsurgical reconstruction of complex trauma of the extremities. Plast Reconstr Surg 1986;78:28592.

8. Boyd LC, Bond GA, Hamidian Jahromi A, et al. Microvascular reconstruction of pediatric lower extremity trauma using free tissue transfer. Eur J Orthop Surg Traumatol 2019; 29:285-93. 
9. Ricci JA, Stranix JT, Lee ZH, et al. Comparing reconstructive outcomes in patients with Gustilo Type IIIB fractures and concomitant arterial injuries. Plast Reconstr Surg 2019; 143:1522-9.

10. Higgins TF, Klatt JB, Beals TC. Lower Extremity Assessment Project (LEAP): the best available evidence on limbthreatening lower extremity trauma. Orthop Clin North Am 2010;41:233-9.

11. Ly TV, Travison TG, Castillo RC, et al. Ability of lower-extremity injury severity scores to predict functional outcome after limb salvage. J Bone Joint Surg Am 2008;90:1738-43.

12. Johansen K, Daines M, Howey T, et al. Objective criteria accurately predict amputation following lower extremity trauma. J Trauma 1990;30:568-73.

13. Howe HR Jr, Poole GV Jr, Hansen KJ, et al. Salvage of lower extremities following combined orthopedic and vascular trauma: a predictive salvage index. Am Surg 1987;53:205-8.

14. McNamara MG, Heckman JD, Corley FG. Severe open fractures of the lower extremity: a retrospective evaluation of the Mangled Extremity Severity Score (MESS). J Orthop Trauma 1994;8:81-7.

15. Russell WL, Sailors DM, Whittle TB, et al. Limb salvage versus traumatic amputation: a decision based on a sevenpart predictive index. Ann Surg 1991;213:473-81.

16. Krettek C, Seekamp A, Kontopp H, et al. Hannover Fracture Scale '98: re-evaluation and new perspectives of an established extremity salvage score. Injury 2001;32:317-28.

17. Macedo JLS, Rosa SC, Botelho DL, et al. Lower extremity reconstruction: epidemiology, management and outcomes of patients of the Federal District North Wing Regional Hospital. Rev Col Bras Cir 2017;44:9-16.
18. Rezende RB, Macedo JL, Rosa SC, et al. Epidemiological profile and treatment of substance losses by trauma to the lower limbs. Rev Col Bras Cir 2017;44:444-51.

19. Lee ZH, Stranix JT, Rifkin WJ, et al. Timing of microsurgical reconstruction in lower extremity trauma: an update of the Godina paradigm. Plast Reconstr Surg 2019;144:759-67.

20. Stranix JT, Rifkin WJ, Lee ZH, et al. Comparison of handsewn versus coupled venous anastomoses in traumatic lower extremity reconstruction. J Reconstr Microsurg 2019;35: 31-6.

21. Stranix JT, Borab ZM, Rifkin WJ, et al. Proximal versus distal recipient vessels in lower extremity reconstruction: a retrospective series and systematic review. J Reconstr Microsurg 2018;34:334-40.

22. Parry SW, Toth BA, Elliott LF. Microvascular free-tissue transfer in children. Plast Reconstr Surg 1988;81:838-40.

23. Upton J, Guo L. Pediatric free tissue transfer: a 29-year experience with 433 transfers. Plast Reconstr Surg 2008;121: 1725-37.

24. Egeler SA, de Jong T, Luijsterburg AJ, et al. Long-term patient-reported outcomes following free flap lower extremity reconstruction for traumatic injuries. Plast Reconstr Surg 2018;141:773-83.

25. Xiong L, Gazyakan E, Kraemer T, et al. Free flaps for reconstruction of soft tissue defects in lower extremity: a metaanalysis on microsurgical outcome and safety. Microsurgery 2016;36:511-24.

26. Arnez ZM, Hanel DP. Free tissue transfer for reconstruction of traumatic limb injuries in children. Microsurgery 1991; 12:207-15. 